\title{
Managed-Care-Ärztenetze: Rückblick auf 2008 und Ausblick aufs neue Jahr
}

\author{
Am 11. Managed-Care-Zukunftsworkshop vom 11. bis 13. Dezember 2008 in \\ Scuol trafen sich erneut verschiedene Vertreter von MC-Ärztenetzen. Zudem \\ nahmen wiederum Vertreter des Dachverbandes med-swiss.net, der SGAM, \\ eines Versicherers und von Santésuisse am jährlichen Gedankenaustausch teil. \\ Am Treffen wurde Bilanz gezogen über die Aktivitäten 2008 der Schweizer \\ Managed-Care-Szene.
}

Urs Keller

Korrespondenz:

Dr. med. Urs Keller

Facharzt für Allgemeinmedizin FMH Büntenstrasse 6

CH-7323 Wangs

urs.keller@hin.ch
$\mathrm{Zu}$ Beginn dieses elften Treffens in Scuol wurde Rückblick gehalten, was im MC-Bereich im Jahr 2008 an Positivem oder Negativem aufgefallen war.

Gut sei im letzten Jahr gewesen, dass es doch Versicherer gab, die viel in MC investierten, dass die Anstrengungen der Netze betreffend Qualitätsmanagement und deren Bedeutung zunehmend wahrgenommen werden und dass gewisse Versicherer diese Anstrengungen schätzen und honorieren. Länger war die Liste der negativen Punkte, u.a. dass die Managed-Care-Szene unverändert heterogen sei und praktisch nicht wahrgenommen werde, ja im Gegenteil, aktuell sei sie wahrscheinlich schwächer als vor zwei Jahren. Sehr negativ beurteilt wurde auch, dass viele Versicherer mehr Anstrengungen in die Gründung von Billigkassen und die Risikoselektion von Versicherten investieren als in die Entwicklung von MC-Modellen. Kritisiert wurde auch, dass die evidenzbasierten Vorteile von MC noch nie untersucht und dargestellt wurden. Auch gibt es kein einheitliches Konzept davon, was man eigentlich unter «MC» versteht. MC wurde zu einem Markt und die Netzwerke tun sich schwer, mit dem Markt umzugehen, ja teilweise verstehen sie den Markt überhaupt nicht.

\section{DRG und Qualität, Bedeutung für MC}

In 2 Grundsatzreferaten wurden Grundlagen über DRG vermittelt, wie es in Deutschland bisher gehandhabt wird und wie die Schweiz die Fehler von Deutschland vermeiden könnte. Zudem wurde die Bedeutung für die Grundversorgung und Praxismedizin diskutiert. Ein Chefarzt und Spitaldirektor gab einen Überblick über das Thema: Wieviel Unternehmertum erträgt das Gesundheitswesen? Er berichtete u.a. über das Konzept des peripheren Kleinspitals Scuol, das auf die Bedürfnisse des Marktes reagierte und verschiedene neue Angebote einführte, von 4 Betten für Komplementärmedizin bis hin zu einem teleradiologisch geführten 24-Stunden-CT. Auch die Gefahren der Regulierungen durch QS-Konzepte und Case-Load-Diskussionen wurden hinterfragt und sehr kritisch diskutiert. Einig war man sich, dass auch in diesem Zusammenhang MC Effizienzsteigerungen ermöglichen soll, dass aktuell und bei der vorgesehenen DRG-Regelung die Anreize falsch gesetzt sind.

Grundsätzlich wurde erneut gefordert, dass gute Qualitätsarbeit durch zusätzliche Abgeltung vergütet werden soll, dass die Attraktivitätssteigerung der MC-Modelle durch Schaffen eines sichtbaren Mehrwertes für den Patienten erfolgen muss und dass in unseren Standesorganisationen die MC-Szene nicht nachhaltig vertreten wird.

\section{Förderung von Managed-Care-Modellen}

Es wurde ein Grundlagenpapier vorgelegt und intensiv diskutiert. Vor allem die unverhandelbare Eckwerte wurden definiert, wie z.B. dass zwingend ein Vertrag zwischen Versicherern und Netzen abgeschlossen werden muss. Zudem wurde längere Zeit kontrovers diskutiert was im Gesetz festgeschrieben werden soll und was nicht, v.a. angesichts der Heterogenität der Ärzteschaft, der Versicherer und der Ansprüche der Patienten. Die Vertreter der Ärztenetze konnten sich schliesslich auf das folgende Positionspapier einigen:

\section{Positionspapier 2008 zu Managed Care}

Die Teilnehmerinnen und Teilnehmer des 11. Managed-Care-Zukunftsworkshops 2008 in Scuol nehmen zur KVG-Reform mit dem Ziel, die Managed-Care-Modelle zu fördern, wie folgt Stellung: 


\section{Präambel}

Basierend auf der Charta für ärztliche Berufsethik [1] gilt auch für uns das Primat des Patientenwohls. Dieses Prinzip verpflichtet grundsätzlich, den Interessen des Patienten zu dienen. Im Mittelpunkt steht das auf gegenseitigem Vertrauen basierende Arzt-Patienten-Verhältnis. Ökonomische Interessen, gesellschaftlicher Druck und administrative Anforderungen dürfen dieses Prinzip grundsätzlich nicht unterlaufen. Trotz Berücksichtigung individueller Bedürfnisse der Patienten müssen Ärzte aber eine Gesundheitsversorgung anbieten, die auf einem klugen und effektiven Einsatz der begrenzten Mittel beruht.

Voraussetzung für die Zukunft von Managed Care in der Schweiz ist ein verbesserter Risikoausgleich.

\section{Eckwerte für sinnvolle und nachhaltige Managed-Care-Modelle (MC)}

1. MC-Vertragspflicht: In den MC-Modellen muss zwingend ein Vertrag zwischen Versicherern und Ärzte-/Versorgungsnetzwerk (Gruppe von Leistungserbringern) abgeschlossen werden (Verträge mit einzelnen Ärzten nur in Ausnahmesituationen); damit soll die weitere Ausbreitung von Pseudo-MCModellen eingedämmt werden.

2. Steuerung: In den MC-Modellen ist ein Betreuungsarzt oder ein ärztlich geführtes Betreuungsteam erste Anlaufstelle für den Patienten. Diese koordinieren alle notwendigen Abklärungen und Behandlungen und stellen die Beziehungen und Koordination der an der Behandlung des Patienten beteiligten Leistungserbringer sicher.

3. Leistungen, Kosten und Qualität: Die Modalitäten der Zusammenarbeit und Vergütungen werden in Verträgen zwischen Ärztenetzen und Versicherern vereinbart.

4. Qualität: Die Ärztenetze definieren und unterhalten ein Qualitätskonzept mit entsprechenden Qualitätsindikatoren und geben Transparenz über die erreichten Ergebnisse. Die Vertragspartner (Ärztenetze - Versicherer) verhandeln darauf aufbauend die entsprechenden Qualitätssicherungsmassnahmen und legen deren Vergütung fest.

5. Erweitertes Leistungsangebot: Im Rahmen eines MC-Vertrages kann die Übernahme von Nichtpflichtleistungen vorgesehen werden.

6. Freiwilligkeit: Die Freiwilligkeit der Versicherten und der Leistungserbringer für den Beitritt in ein MC-Modell oder der Austritt muss gewährleistet sein. Längerfristige Verträge für Versicherte (länger als ein Jahr) sollen möglich sein.
7. Organisation: Die Ärzte-/Versorgungsnetze organisieren sich autonom. Das Netzwerk regelt die Zusammenarbeit mit Spezialisten und weiteren Leistungserbringern.

8. Budgetmitverantwortung: Diese kann zwischen den Vertragspartnern (Ärztenetzwerk Versicherer) frei verhandelt werden.

9. Einsparungen: Durch Managed Care erzielte Einsparungen (inkl. logistische Einsparungen) verbleiben in jedem Fall im System des Managed-Care-Vertrages.

10. Anreize: Versicherte in MC-Modellen sollen gegenüber konventionell Versicherten begünstigt werden (Mehrwert, finanzielle Vorteile, Dienstleistungen, insbesondere soll ein differenzierter Selbstbehalt in Betracht gezogen werden).

\section{Konsequenzen für die Gesetzgebung}

- Verbesserter Risikoausgleich unter den Krankenversichern;

- Managed-Care-Modelle beruhen auf einem Vertrag zwischen den Partnern;

- differenzierter Selbstbehalt; mehr Gestaltungsspielraum bei der Regelung des Selbstbehalts;

- in Managed-Care-Modellen können auch Nichtpflichtleistungen vergütet werden (Ausweitung des Leistungskataloges);

- Möglichkeit der Verlängerung der Vertragsdauer über ein Jahr hinaus.

\section{Diskussionen}

Auch die SGAM-Position, insbesondere zur dualen Grundversicherung, und die Position des MC-Dachverbandes med-swiss.net zur KVGGrundversicherungsreform mit dem Ziel, die

\section{Teilnehmer des Workshops}

vom 11. bis 13. Dezember 2008 in Scuol Dres. med. Roman Buff (vmco, thurcare), Wolfgang Czerwenka (argomed), Margot Enz Kuhn (SGAM), Jörg Fritschi (medswiss.net), Donat Gemsch (mednetbern), Felix Huber (mediX Zürich), Rainer Hurni (zmed), Urs Keller (PizolCare), Stephan Koch (Netz Freiamt), Konrad Schiess (xundart), Michael Siegenthaler (Murgmed), Max von Salis (HAPmed); Dr. med. Jürg Vontobel (concordia), lic. oec. Stefan Kaufmann (santésuisse). Marcel Marolf und Rudolf Wartmann (Novartis Pharma Schweiz AG) Der Workshop wurde unterstützt von der Firma Novartis Pharma Schweiz AG und moderiert von Dr. H. J. Schlegel. 
Managed-Care-Modelle zu fördern und Lösungen als Ersatz für den Zulassungsstopp festzulegen, wurden längere Zeit kontrovers diskutiert. Aus Sicht von santésuisse und den Versicherern wurde betont, dass die gesetzlichen Einschränkungen nicht zu rigide formuliert werden dürfen, sondern möglichst offen gehalten werden sollen, um grösstmögliche Freiheiten für Vertragsverhandlungen zu bewahren.

Vorgestellt wurde auch, wie die Hausarztausbildung attraktiv gefördert werden kann, wie zukünftige Praxisformen aussehen werden, wie neue Netze entstehen und sich grosse Betriebsgesellschaften reorganisieren und wie Netzwerke die Gesundheitsförderung in Betrieben unterstützen können.

\section{Fazit}

An den drei Tagen im tief verschneiten Scuol wurde, weit weg vom Alltag, erneut eine produktive und offene Diskussion ohne Vorurteile unter den MC-Verantwortlichen der Deutschschweiz zusammen mit Krankenkassenvertretern geführt. Es wurde Bilanz gezogen, die dieses Jahr deutlich skeptischer ausfiel. Es passte dazu, dass v. a. gesundheitspolitische Probleme rund um DRG, KVG-Reform, Förderung von MC und neue Praxisformen im Zentrum der Diskussionen standen.
Definition eines Netzwerkes (med-swiss.net) Ärztenetzwerke bzw. Versorgungsnetze sind Organisationen, die von Leistungserbringern gebildet werden und der Gesundheitsversorgung dienen. Durch verbindliches Zusammenwirken unter sich, mit netzfremden Leistungserbringern und mit den Kostenträgern erbringen sie auf die Bedürfnisse der Patientinnen und Patienten ausgerichtete Gesundheitsleistungen. Dieses Zusammenwirken beruht auf vereinbarten Behandlungsprozessen, unternehmerischen Organisationsstrukturen und einer gemeinsamen Betreuungskultur. Sie setzen sich bei hoher Versorgungsqualität einen haushälterischen Umgang mit den vorhandenen Mitteln zum Ziel. Ein weiteres Merkmal der Versorgungsnetze ist der Wille, die Gesundheitsleistungen aus einer Hand zu steuern.

\section{Literatur}

1 ABIM Foundation. American Board of Internal Medicine; ACP-ASIM Foundation. American College of Physicians-American Society of Internal Medicine; European Federation of Internal Medicine. Medical professionalism in the new millennium: a physician charter. Ann Intern Med. 2002;136(3):243-6. 